\section{Profile of depressive symptoms in sleep apnea- gender differences and the role of obesity}

\section{Abstract}

Objective: The objective was to evaluate depressive symptoms, sleep alterations and the role of gender and obesity in obstructive sleep apnea (OSA) patients.

Methods: Patients ( $\mathrm{N}=140 ; 92$ males and 48 female; age 54.6 \pm 8.2 years), submitted to polysomnography for suspected OSA, were assessed by the 17-item Hamilton Rating Scale for Depression (HRSD), Charlson comorbidity index (CCI) and Epworth Sleepiness Scale (ESS). All patients were divided in two groups: snorers/mild OSA group (apnea hypopnea index $(\mathrm{AHI}) \leq 15, \mathrm{~N}=54$ ) and moderate/severe OSA group $(\mathrm{AHI}>15, \mathrm{~N}=86)$.

Results: The most affected components of the HDRS in both genders were anxiety, somatic and psychological, followed by work and activities complaints and depressed mood. Late insomnia predominated over early and middle night insomnia. Snorers/mild OSA women showed higher HDRS scores $(p=0.002)$. Obese patients showed higher HDRS scores vs non-obese (BMl>30, $\mathrm{N}=96)(8.52 \pm 5.0$ vs $6.38 \pm 5.0$, $p=0.02$ ). The profile of depressive symptoms was similar for obese vs non-obese. Excessive daytime sleepiness (ESS>10) was present in 57 cases (40.7\%). ESS scores were negatively correlated with minimum $\mathrm{SpO}_{2}$ values $(r=-0.18, p=0.03)$ and positively correlated with arousal frequency $(r=0.24, p=0.02)$. Sedatives $(12.1 \%)$ or antidepressants (5.7\%) were seldom used. Conclusion: Depressive symptoms in OSA, in both genders, are characterized by somatic and psychological anxiety, work complaints, depressed mood and late insomnia; obese patients are more affected.
Pablo Antonio Maia de Farias ${ }^{1}$, Camila Andrade Mendes Medeiros', Marcela Lima Gurgel2 ${ }^{2}$, Cauby Maia Chaves-Junior ${ }^{3}$, Pedro Felipe Carvalhedo de Bruin ${ }^{4}$, Veralice Meireles Sales de Bruin4

$1 \mathrm{PhD}$, Pharmacist, Universidade Federal do Ceará, Brazil.

2 Master's Degree Student, Universidade Federal do Ceará, Brazil.

3 PhD, Associate Professor, Universidade Federal do Ceará, Brazil.

$4 \mathrm{MD}, \mathrm{PhD}$, Associate Professor, Department of Medicine, Universidade Federal do Ceará, Brazil

Contact information:

Veralice Meireles Sales de Bruin.

”v veralicebruin@gmail.com

Keywords

sleep apnea; anxiety; depression; sleepiness; polysomnography; obesity. 


\section{Introduction}

Obstructive sleep apnea (OSA) is an important clinical condition which is believed to affect at least $10 \%$ of the population, leading to high morbidity and mortality ${ }^{1,2}$. Although depressive symptoms have been frequently described in association with OSA, a causal relationship between these two conditions is still under dispute ${ }^{3,4}$. It has been shown that patients with major depressive disorders and post-traumatic stress are at increased risk of OSA ${ }^{5}$. Recently, a study has suggested that fatigue predicts depressive symptoms in patients with OSA ${ }^{6}$. This poses the important issue that depressive symptoms might be merely secondary to reduced welfare associated with fatigue. It must be kept in mind that psychiatric comorbidity in OSA may also affect patient quality of life and adherence to continuous positive airway pressure (CPAP).

Gender differences are important to guide therapy. Furthermore, the role of obesity in the genesis of depressive symptoms have not been fully appreciated in OSA. It has been shown that women tend to be under-diagnosed for OSA due to atypical symptoms or different clinical presentation. Overweight was associated with incident depression in women, while obesity were associated with incident depression in both genders ${ }^{7,8}$. Women with OSA have been reported to weigh more, have more complaints of insomnia ${ }^{9}$, be more depressed and seek medical attention at an older age ${ }^{8}$. Thus, previous evidences indicate that gender differences related to OSA, sleep alterations and depressive symptoms exist and these may be important for clinical management.

Previous studies indicate that obesity, sleep alterations, depression and metabolic syndrome are connected $^{10-12}$. It has been shown that short sleep contributes to weight gain and obesity ${ }^{13}$. Excessive daytime sleepiness (EDS) has also been linked to depression in the obese ${ }^{14}$. Among adolescents, anxiety and depressive symptoms are positively associated with increased body mass index (BMI) and body fat ${ }^{15,16}$. Moreover, depression and anxiety have been linked to adverse health behaviors possibly interfering with daily activities and sleep hygiene $^{17}$. Thus, psychological distress seems to be an important determinant of sleep quality and excessive daytime sleepiness in obese patients. It has been proposed that the presence/absence of psychological distress could provide the basis for understanding the different phenotypes related to obesity. In other words, it could help to explain why some individuals present poor sleep, fatigue and hyperactivity and others show better sleep and excessive daytime sleepiness ${ }^{10}$. All this confirm the close relationship between sleep alterations, obesity and anxiety/depression. Understanding the profile of these symptoms may guide disease modifying strategies.

The aim of this study was evaluate depressive symptoms, sleep alterations and the role of gender and obesity in obstructive sleep apnea (OSA) patients.

\section{Methods}

\section{Study population}

This is a cross-sectional evaluation of 150 consecutive patients referred for polysomnography with clinical suspicion of OSA. Cases with cancer, severe neurological, renal, hepatic, lung or cardiac diseases were excluded. Five individuals declined to participate in the study and five other were considered too ill to participate. Among the latter, three had dementia and two had suffered a recent ischemic stroke. Thus, the final sample consisted of 140 patients. After overnight polysomnography, those with Apnea-Hypopnea Index (AHI) $\leq 5$ events per hour of sleep were diagnosed as snorers, $5<\mathrm{AHI}<15$ as mild, $15<A H I<30$ as moderate and those with AHI $>30$ as severe OSA. For comparison, all cases were grouped as snorers/mild OSA $(A H I \leq 15)$ and as moderate/severe OSA (AHI>15). Cases included 
in the study were not involved in shift work and did not have recent hospitalizations for the last three months. A structured interview was conducted prior to polysomnography. The protocol was approved by the local Research Ethics Committee (COMEPE 093.12.08) and written informed consent was obtained in all cases.

\section{Procedures}

Demographic and clinical data were recorded using a closed-question data collection instrument obtained in a face to face interview. Body mass index was calculated as the ratio between weight $(\mathrm{Kg})$ and squared height $\left(\mathrm{m}^{2}\right)$. A special emphasis was made on the use of medication in the last 30 days.

Depressive symptoms were evaluated by the Hamilton Rating Scale for Depression-17 item (HRSD) ${ }^{18}$. This scale takes into consideration several aspects such as anxiety (psychological and somatic), depressed mood, insomnia subdivided in early, middle and late insomnia among other mood related questionings. Daytime somnolence was assessed by the Epworth sleepiness scale (ESS), a validated questionnaire containing eight items that asks for expectation of dozing in eight hypothetical situations. Dozing probability ratings range from zero (no probability) to three (high probability). An ESS score of 10 or more indicates excessive daytime sleepiness $^{19}$. Comorbidity severity was evaluated with the Charlson Comorbidity Index $(\mathrm{CCl})^{20}$.

Standard overnight polysomnography was performed on a digital polygraph (ALICE $\| ®$, Respironics Inc.). Polysomnographic recordings were set to begin at 10 p.m. (lights-out) and end at 6 a.m. (lights-on). Monitored variables included: electroencephalogram $(\mathrm{C} 3, \mathrm{C} 4, \mathrm{O} 1, \mathrm{O} 2$ referenced to contralateral ear electrodes), bilateral electrooculograms, submental electromyogram (EMG), two-lead electrocardiogram, pulse oximetry, bilateral tibialis EMG and airflow, using a nasal/oral thermocouple. Body position and thoracic and abdominal movements (inductance plethysmography) were also recorded.
Sleep staging was performed by 30 -s epochs, according to standard procedures ${ }^{21}$. Polysomnographyderived parameters evaluated were $\mathrm{AHI}$, minimum oxygen saturation $\left(\mathrm{SaO}_{2} \mathrm{~min}\right)$, sleep latency, sleep efficiency, rapid eye movement (REM) sleep latency, amount of REM sleep (\% of total sleep time), amount of non-rapid eye movement (NREM) sleep (\% of total sleep time), number of arousals and periodic leg movements. Arousal analysis and scoring of respiratory events during sleep were performed according to standard criteria. Apneas were defined as cessation of airflow for $10 \mathrm{~s}$ or more and hypopneas as a reduction of inspiratory air flow of $50 \%$ or more associated with either oxygen desaturation of $>3 \%$ or an arousal.

\section{Statistical analysis}

Analyses were carried out by the Statistical Package for Social Sciences V16.0 [SPSS Inc, Chicago (IL), USA]. For normally distributed variables with homogeneity of variance, we performed two tailed Student test. For those variables that did not meet the homogeneity of variances requirement, we used nonparametric Mann-Whitney $U$ test and for categorical variables, Fisher's exact test. Spearman correlation test was used to verify linear associations. Differences between groups were considered to be statistically significant at $p<0.05$.

\section{Results}

One hundred and forty patients of both genders, $92(65,7 \%)$ male and 48 (34,3\%) female, aged 19 to 81 years (mean age $54.6 \pm 8.2$ years) were included in the study. Eighty-six cases (61.4\%) were classified as moderate/severe OSA and 54 (38.6\%) as snorers/mild OSA. Moderate/severe OSA cases were predominantly of male gender (65 men vs 21 women, $\mathrm{p}<0.005$ ).

Table 1 shows clinical, demographic and polysomnographic data according to gender. Women 
that were snorers/mild OSA had more severe depressive symptoms (HDRS scores, $p=0.002$ ). Women with moderate/severe OSA showed greater comorbidity severity (CIRS scores, $p=0.04$ ). For both genders, cardiovascular complaints were the main determinants of the $\mathrm{CCl}$. Considering both genders, HDRS scores $(7.69 \pm 5.5$ vs $6.65 \pm 4.8 ; \mathrm{p}=0.37)$ and ESS scores $(9.24 \pm 4.3$ vs $10.45 \pm 4.6 ; p=0.15)$ were not different between snorers/mild OSA and mo- derate/severe OSA, respectively.

Table 2 depicts matrix correlations between clinical and polysomnographic variables among all cases. Age was negatively correlated with sleep efficiency $(r=-0.33, p=0.000)$ and with lowest nocturnal oxygen saturation $(r=-0.177, p=0.04)$. Body mass index was positively correlated with $\mathrm{AHI}(r=0.30$, $p=0.000)$ and arousals $(r=0.22, p=0.03)$ and negatively correlated with oxygen saturation values $(r=-$

Table 1. Clinical and polysomnographic characteristics of patients grouped according to gender and by apnea+hypopnea index severity

\begin{tabular}{|c|c|c|c|c|c|}
\hline Variables & \multicolumn{2}{|c|}{$\begin{array}{l}\text { Male } \\
\mathrm{N}=92\end{array}$} & \multicolumn{3}{|c|}{$\begin{array}{l}\text { Female } \\
\mathrm{N}=48\end{array}$} \\
\hline OSA & $\begin{array}{l}\text { Snorers/mild } \\
\qquad \begin{array}{c}\mid A H \leq 15 \\
N=27\end{array}\end{array}$ & $\begin{array}{c}\text { Moderate/severe } \\
\qquad \begin{array}{c}\mathrm{A} H>15 \\
\mathrm{~N}=65\end{array}\end{array}$ & $\begin{array}{c}\text { Snorers/mild } \\
\qquad \begin{array}{c}\mid A H \leq 15 \\
N=27\end{array}\end{array}$ & $\begin{array}{c}\text { Moderate/severe } \\
\qquad \begin{array}{c}\mathrm{A} H \\
\mathrm{~N}=21\end{array}\end{array}$ & $\begin{array}{l}\text { P value } \\
\text { G1 vs } G 3 \\
\text { G2 vs } G 4\end{array}$ \\
\hline Age (y) & $36.9(13.2)$ & $48.0(15.1)$ & $42.2(11.3)$ & $49.7(13.4)$ & $\begin{array}{l}\text { a } 0.12 \\
0.65\end{array}$ \\
\hline BMI $\left(\mathrm{Kg} / \mathrm{m}^{2}\right)$ & $26.4(3.5)$ & $30.4(6.3)$ & $27.3(5.4)$ & $29.3(6.2)$ & $\begin{array}{l}\text { a } 0.44 \\
0.48\end{array}$ \\
\hline HDRS (scores) & $5.3(3.3)$ & $6.2(4.4)$ & $10.0(6.3)$ & $8.0(5.7)$ & $\begin{array}{c}\mathrm{b} 0.002 * \\
0.14\end{array}$ \\
\hline ESS (scores) & $9.4(4.5)$ & $10.2(4.5)$ & $9.0(4.2)$ & $11.0(4.9)$ & $\begin{array}{l}\mathrm{b} 0.78 \\
0.53\end{array}$ \\
\hline CCI (scores) & $2.9(1.7)$ & $3.4(1.9)$ & $3.6(2.4)$ & $4.3(1.5)$ & $\begin{array}{l}\text { b } 0.23 \\
0.04^{\star}\end{array}$ \\
\hline $\begin{array}{l}\text { Sleep efficiency } \\
(\%)\end{array}$ & $87.6(10.8)$ & $82.5(11.5)$ & $90.9(7.3)$ & $85.5(9.7)$ & $\begin{array}{l}\text { a } 0.19 \\
0.28\end{array}$ \\
\hline $\begin{array}{l}\text { Sleep latency } \\
\text { (min) }\end{array}$ & $9.1(7.1)$ & $13.1(10.9)$ & $12.7(11.6)$ & $15.7(18.5)$ & $\begin{array}{l}\text { a } 0.17 \\
0.06\end{array}$ \\
\hline $\begin{array}{l}\text { REM latency } \\
\text { (min) }\end{array}$ & $102.8(49.5)$ & $126.2(70.3)$ & $108.6(76.4)$ & $94.6(49.8)$ & $\begin{array}{l}0.70 \\
0.06\end{array}$ \\
\hline AHI & $8.6(3.4)$ & $41.6(21.7)$ & $6.5(4.0)$ & $32.9(19.2)$ & $\begin{array}{l}0.04^{\star} \\
0.10\end{array}$ \\
\hline SpO2min & $85.9(3.4)$ & $77.4(10.4)$ & $86.2(4.3)$ & $79.1(9.0)$ & $\begin{array}{l}\text { a } 0.77 \\
0.51\end{array}$ \\
\hline $\begin{array}{l}\text { Time with } \\
\text { SpO2<90\% (min) }\end{array}$ & $26.5(58.3)$ & $66.9(92.4)$ & $2.2(1.9)$ & $35.6(42.8)$ & $\begin{array}{l}\text { a } 0.38 \\
0.11\end{array}$ \\
\hline $\begin{array}{l}\text { Arousals } \\
\text { (events/h) }\end{array}$ & $23.4(9.8)$ & $35.1(18.7)$ & $18.5(8.7)$ & 25.9 (11.9) & $\begin{array}{l}0.18 \\
0.21\end{array}$ \\
\hline
\end{tabular}

Data are presented as mean (SD) values and frequency (\%) values.

* $P<0.05$ Student's t test

b Mann-Whitney test

Abbreviations: BMI= Body Mass Index; HRSD= Hamilton Rating Scale for Depression; ESS= Epworth Sleepiness Scale;

Charlson Comorbidity Index=CCl; $\mathrm{AHI}=$ Apnea+Hypopnea Index; $\mathrm{SpO}_{2}$ min= Minimal peripheral oxygen saturation 
Table 2. Matrix correlations (Spearman) between clinical and polysomnographic variables in patients with obstructive sleep apnea syndrome

\begin{tabular}{|c|c|c|c|c|}
\hline Variables & Age & BMI & $\begin{array}{l}\text { HDRS } \\
\text { scores }\end{array}$ & $\begin{array}{c}\text { ESS } \\
\text { Scores }\end{array}$ \\
\hline Sleep efficiency & $\begin{array}{l}r=-0.33 \\
p=0.000 * *\end{array}$ & $\begin{array}{l}r=0.10 \\
p=0.21\end{array}$ & $\begin{array}{l}r=0.07 \\
p=0.38\end{array}$ & $\begin{array}{l}r=0.03 \\
p=0.66\end{array}$ \\
\hline Sleep latency & $\begin{array}{l}r=0.12 \\
p=0.14\end{array}$ & $\begin{array}{l}r=0.03 \\
p=0.72\end{array}$ & $\begin{array}{l}r=0.03 \\
p=0.66\end{array}$ & $\begin{array}{l}r=-0.01 \\
p=0.90\end{array}$ \\
\hline REM latency & $\begin{array}{l}r=-0.007 \\
p=0.93\end{array}$ & $\begin{array}{l}r=-0.01 \\
p=0.85\end{array}$ & $\begin{array}{l}r=0.11 \\
p=0.17\end{array}$ & $\begin{array}{l}r=-0.14 \\
p=0.09\end{array}$ \\
\hline REM amount & $\begin{array}{l}r=-0.03 \\
p=0.79\end{array}$ & $\begin{array}{l}r=0.07 \\
p=0.50\end{array}$ & $\begin{array}{l}r=-0.10 \\
p=0.35\end{array}$ & $\begin{array}{l}r=0.09 \\
p=0.39\end{array}$ \\
\hline Apnea+hypopnea index & $\begin{array}{l}r=0.139 \\
p=0.10\end{array}$ & $\begin{array}{l}r=0.300 \\
p=0.000 * *\end{array}$ & $\begin{array}{l}r=-0.027 \\
p=0.75\end{array}$ & $\begin{array}{l}r=0.065 \\
p=0.44\end{array}$ \\
\hline $\mathrm{SpO}_{2} \min$ & $\begin{array}{l}r=-0.177 \\
p=0.04^{*}\end{array}$ & $\begin{array}{l}r=-0.395 \\
p=0.000 * *\end{array}$ & $\begin{array}{l}r=0.104 \\
p=0.23\end{array}$ & $\begin{array}{l}r=-0.18 \\
p=0.03 *\end{array}$ \\
\hline Arousals index & $\begin{array}{l}r=-0.12 \\
p=0.27\end{array}$ & $\begin{array}{l}r=0.22 \\
p=0.03 *\end{array}$ & $\begin{array}{l}r=-0.17 \\
p=0.10\end{array}$ & $\begin{array}{l}r=0.24 \\
p=0.02 *\end{array}$ \\
\hline
\end{tabular}

Abbreviations: $\mathrm{BMI}=$ Body Mass Index; HRSD = Hamilton Rating Scale for Depression; ESS= Epworth Sleepiness Scale;

$\mathrm{AHI}=\mathrm{SpO}_{2} \mathrm{~min}=$ Minimal oxygen saturation; $\mathrm{REM}=$ Rapid eye movement; $\mathrm{SpO}_{2} \mathrm{~min}=$ Minimal peripheral oxygen saturation

0.395, $p=0.000)$. Epworth Sleepiness Scale scores were negatively correlated with oxygen saturation $(r=-0.18, p=0.03)$ and positively correlated with arousals ( $r=0.24, p=0.02)$.

The analysis of the components of the HDRS showed that the most important complaints are somatic and psychological anxiety, followed by work and activities complaints, late insomnia, somatic symptoms, depressed mood, hypochondriasis and genital symptoms (Figure 1). Among all cases, 105 patients $(73.4 \%)$ were overweight $(25<\mathrm{BMI} \leq 30)$ and 42 (29\%) were obese (BMl>30). In eight cases (5.5\%), BMI was greater than 40 . Obese patients $(\mathrm{N}=96)$ showed higher HDRS scores $(8.52 \pm 5.0$ vs

Figure 1: Components of the Hamilton Depressive Rating Scale in patients with OSAS

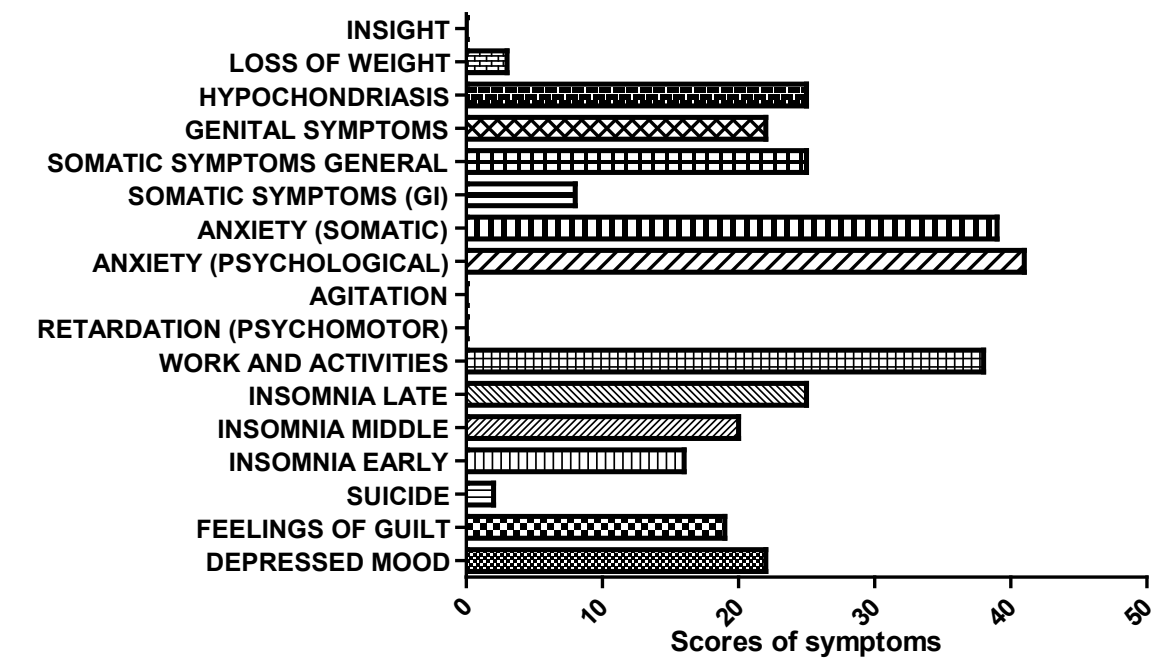


$6.38 \pm 5.0, p=0.02$ ). Profile of depressive symptoms was similar for obese and non-obese patients. In all cases, BMI was positively correlated with HDRS scores $(r=0.246, p=0.02$ ) (Figure 2 ). Seventeen patients $(12.1 \%)$ used sedatives, mostly benzodiazepine, and eight cases (5.7\%) were on antidepressants (selective inhibitors of serotonin reuptake).

\section{Discussion}

There are gender related differences in sleep apnea: obstructive sleep apnea is more common in men $^{22}$. Similarly, in this series of cases and according to OSA severity, moderate/severe OSA predominantly affected men. In the present work, women with mild OSA, had more severe depressive symptoms. In partial agreement, depression was more prevalent in women with OSA than in men ${ }^{23-25}$. This needs attention considering that patients with comorbid conditions such as anxiety and depression are increased risk of cardiovascular disease and mortality ${ }^{26,27}$. Other works confirm that women with OSA may have greater risk for hypertension and endothelial dysfunction ${ }^{28}$.
Depressive symptoms and sleep parameters, i.e. apnea+hypopnea index, $\mathrm{SpO}_{2}$ min and HDRS scores, did not correlate. In corroboration, previous studies showed that depressive symptoms were not correlated with OSA severity ${ }^{24,29}$. In this work, BMI measures correlated with the HDRS scores. Studies involving OSA, obesity, gender and depression are still controversial. Hence, it is important and necessary to clarify the association among these factors for a proper clinical management.

Our results showed a positive correlation among the BMI, apnea+hypopnea index, arousals index and $\mathrm{SpO}_{2}$ min. Furthermore, obesity was associated with more severe depressive symptoms. In agreement with a previous study, patients with OSA, overweight and obesity had more depression ${ }^{30}$.

Given the present evidence and considering the high prevalence of obesity, depression and poor quality of life in OSA patients, probably, a multitherapy approach to treat obesity, OSA and depressive symptoms is necessary. For instance, a survey that evaluated assessment of bariatric surgery efficacy on OSA determined a significant improvement in AHI/ $\mathrm{Al} / \mathrm{RDI}$ occurred after surgery, in addition to the foreseeable reduction in body mass index ${ }^{31}$. Respiratory

Figure 2: Body mass index is directly correlated with HDRS scores

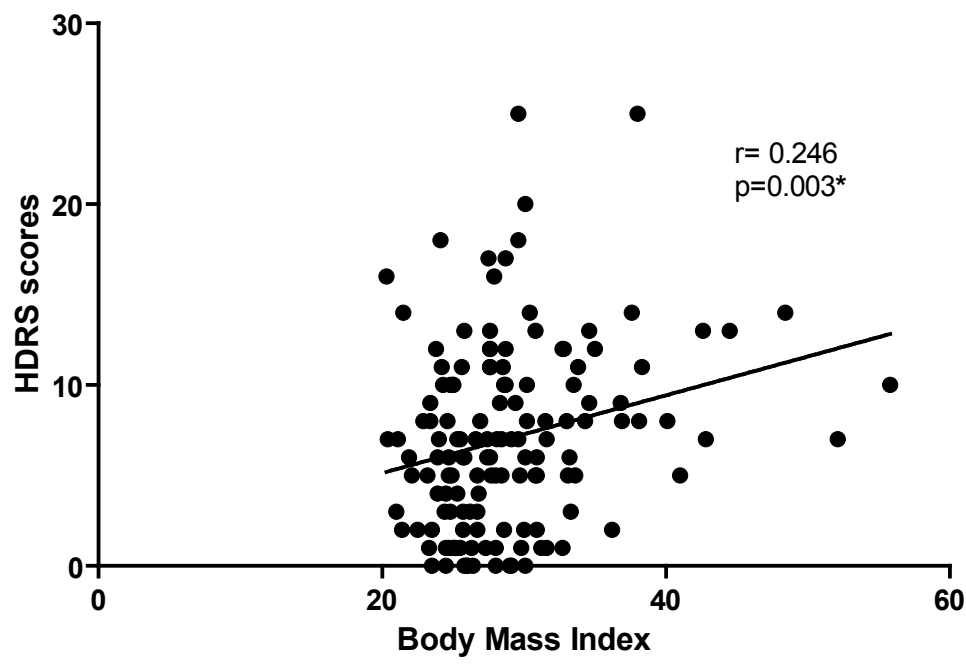


symptoms in individuals with OSA can be treated with components as continuous positive airway pressure (CPAP) $)^{32,33}$ or mandibular advancement device (MADs) ${ }^{32}$. Furthermore, evaluating depressive symptoms is necessary before proceeding with non-pharmacological and/or pharmacological support.

Presently, patients with OSA presented some specific psychological characters of mood symptoms. Somatic and psychological anxiety followed by work and activities complaints, were the most important depressive symptoms domains in OSA patients. Importantly, late insomnia was predominant over middle and early insomnia. Similarly, others studies reported a strong association between OSA and somatic syndromes, anxiety and insomnia ${ }^{34-37}$. Women, habitual snorers or with mild OSA, presented more somatic anxiety: obese patients had more severe symptoms. These findings reinforce the importance of gender and obesity in the manifestation of depressive symptoms. The role of anxiety in the determination of mood symptoms as presented here is relevant considering that a recent study demonstrated that depression and anxiety were associated with decreased health-related quality of life scales $(\mathrm{HRQL})^{33,38}$. Depressive symptoms are also important as they seem to be a major contributor to mental and physical quality of life $\mathrm{e}^{38}$.

Interestingly, ESS scores were correlated with arousals index and SpO2min values. However, sleepiness severity (ESS scores) were not correlated to apnea+hypopnea index. In agreement, polysomnographic nocturnal hypoxaemic parameters were previously correlated with sleepiness measures (ESS scores) $)^{39}$.

In our findings, women with moderate/severe OSA showed greater comorbidity severity than men. According with a study that included a large number of patients with OSA, a strongly association between OSA and significant comorbidities was demonstrated. Type 2 diabetes and ischaemic heart disease were more prevalent in men; however, hypertension was more common in women ${ }^{23}$.
We accuse knowledge limitations. Sleepiness was only assessed by a subjective measure. However, it has been argued that objective and subjective measures of sleepiness represent different constructs. As a positive aspect, ESS is largely used in clinical studies.

\section{Conclusion}

The profile of depressive symptoms in OSA is determined by anxiety, both somatic and psychological, followed by work and activities complaints, late insomnia, somatic symptoms and depressed mood. Depressive symptoms are more severe in women and in obese patients and, in general, are overlooked and undertreated.

\section{Conflict of interest disclosure}

The authors report no conflicts of interest.

\section{Acknowledgements}

This study was partially supported by The Brazilian Council for Scientific Research (CNPq-MCT) and FUNCAP.

\section{References}

1. Young T, Palta M, Dempsey J, Skatrud J, Weber S, Badr S. The occurrence of sleep-disordered breathing among middle-aged adults. N Engl J Med. 1993; 328(17): 1230-5.

2. Tufik S, Santos-Silva R, Taddei JA, Bittencourt LR. Obstructive sleep apnea syndrome in the Sao Paulo Epidemiologic Sleep Study. Sleep Med. 2010; 11(5): 441-6.

3. Aloia MS, Arnedt JT, Smith L, Skrekas J, Stanchina M, Millman RP. Examining the construct of depression in obstructive sleep apnea syndrome. Sleep Med. 2005; 6(2): 115-21.

4. Macey PM, Woo MA, Kumar R, Cross RL, Harper RM. Relationship between obstructive sleep apnea severity and sleep, depression 
and anxiety symptoms in newly-diagnosed patients. PLoS One. 2010; 5(4): 10211

5. Gupta MA, Simpson FC. Obstructive Sleep Apnea and Psychiatric Disorders: A Systematic Review. J Clin Sleep Med. 2014; 19.

6. Jackson ML, Stough C, Howard ME, Spong J, Downey LA, Thompson B. The contribution of fatigue and sleepiness to depression in patients attending the sleep laboratory for evaluation of obstructive sleep apnea. Sleep Breath. 2011; 15(3): 439-45.

7. B Brumpton, A Langhammer, P Romundstad, Y Chen, X-M Mai. The associations of anxiety and depression symptoms with weight change and incident obesity: The HUNT Study. IJO. 2012; 27: 1268-74.

8. Ye L, Pien GW, Weaver TE. Gender differences in the clinical manifestation of obstructive sleep apnea. Sleep Med. 2009; 10(10): 1075-84.

9. Roure N, Mediano O, Duran-Cantolla J, Garcia Rio F, de la Pena $\mathrm{M}$, Capote $\mathrm{F}$, et al. [Differences in clinical and polysomnographic variables between male and female patients with sleep apneahypopnea syndrome]. Arch Bronconeumol. 2008; 44(12): 685-8.

10. Vgontzas AN, Bixler EO, Chrousos GP, Pejovic S. Obesity and sleep disturbances: meaningful sub-typing of obesity. Arch Physiol Biochem. 2008; 114(4): 224-36.

11.Gylen E, Anttalainen U, Saaresranta T. Relationship between habitual sleep duration, obesity and depressive symptoms in patients with sleep apnoea. Obes Res Clin Pract. 2014; 8(5): 459-65.

12. Hayley AC, Williams LJ, Venugopal K, Kennedy GA, Berk M, Pasco JA. The relationships between insomnia, sleep apnoea and depression: Findings from the American National Health and Nutrition Examination Survey. Aust N Z J Psychiatry. 2014; 15

13. Singh $M$, Drake $C L$, Roehrs $T$, Hudgel DW, Roth $T$. The association between obesity and short sleep duration: a population-based study. J Clin Sleep Med. 2005; 1(4): 357-63.

14. Bixler EO, Vgontzas AN, Lin HM, Calhoun SL, Vela-Bueno A, Kales A. Excessive daytime sleepiness in a general population sample: the role of sleep apnea, age, obesity, diabetes, and depression. J Clin Endocrinol Metab. 2005; 90(8): 4510-5.

15. Hillman JB, Dorn LD, Huang B. Association of Anxiety and Depressive Symptoms and Adiposity Among Adolescent Females Using Dual Energy X-ray Absorptiometry. Clin Pediatr (Phila). 2010; 31.

16. Xanthopoulos MS, Gallagher PR, Berkowitz RI, Radcliffe J, Bradford R, Marcus CL. Neurobehavioral Functioning in Adolescents With and Without Obesity and Obstructive Sleep Apnea. Sleep. 2014; 17.

17. Chasens ER, Twerski SR, Yang K, Umlauf MG. Sleepiness and health in midlife women: results of the National Sleep Foundation's 2007 Sleep in America poll. Behav Sleep Med. 2010; 8(3): 157-71.

18. Hamilton M. A rating scale for depression. J Neurol Neurosurg Psychiatry. 1960; 23:56-62
19. Johns MW. A new method for measuring daytime sleepiness: the Epworth sleepiness scale. Sleep. 1991; 14(6): 540-5.

20. Charlson ME, Pompei $P$, Ales KL, MacKenzie CR. A new method of classifying prognostic comorbidity in longitudinal studies: development and validation. J Chronic Dis. 1987; 40(5): 373-83.

21. Miano S, Paolino MC, Castaldo R, Villa MP. Visual scoring of sleep: A comparison between the Rechtschaffen and Kales criteria and the American Academy of Sleep Medicine criteria in a pediatric population with obstructive sleep apnea syndrome. Clin Neurophysiol. 2010; 121(1): 39-42.

22. Franklin KA, Lindberg E. Obstructive sleep apnea is a common disorder in the population - a review on the epidemiology of sleep apnea. J Thorac Dis. 2015; 7(8): 1311-22.

23. Mokhlesi B, Ham SA, Gozal D. The effect of sex and age on the comorbidity burden of OSA: an observational analysis from a large nationwide US health claims database. Eur Respir J. 2016; 47(4): 1162-9.

24. Sforza E, Saint Martin M, Barthélémy JC, Roche F. Mood disorders in healthy elderly with obstructive sleep apnea: a gender effect. Sleep Med. 2016; 19(57) 62.

25. Lee MH, Lee AS, Lee GH, Ryu HS, Chung S, Chung YS, Kim WS, Gender differences in the effect of comorbid insomnia symptom on depression, anxiety, fatigue, and daytime sleepiness in patients with obstructive sleep apnea. Sleep Breath 2014; 18(1): 111-17.

26. Kemp AH, Quintana DS, Quinn CR, Hopkinson P, Harris AW. Major depressive disorder with melancholia displays robust alterations in resting state heart rate and its variability: implications for future morbidity and mortality. Front Psychol. 2014; 27(5): 1387.

27. Lunar CB, Campelo PG, Valladolid JC, Rodríguez CYF, Menéndez MIG, López FL, Fort MAS. Effect of depression on mortality and cardiovascular morbidity in type 2 diabetes mellitus after 3 years follow up. The DIADEMA study protocol. BMC Psychiatry. 2012; 12(95).

28. Won C, Guilleminault C. Gender differences in sleep disordered breathing: implications for therapy. Expert Rev Respir Med 2015; 9(2): 221-31

29. Rezaeitalab F, Moharrari F, Saberi S, Asadpour H, and Rezaeetalab F. The correlation of anxiety and depression with obstructive sleep apnea syndrome. J Res MedSci. 2014; 19(3): 205-10.

30. LaGrotte C, Fernandez-Mendoza J, Calhoun SL, Liao D, Bixler EO, Vgontzas AN. The relative association of obstructive sleep apnea, obesity and excessive daytime sleepiness with incidentdepression: a longitudinal, population-based study. Int J Obes (Lond). 2016; 24.

31. Quintas-Neves M, Preto J, Drummond $M$. Assessment of bariatric surgery efficacy on Obstructive Sleep Apnea (OSA). Rev Port Pneumol. 2016; 20(16): 173-5115

32. Povitz M, Bolo CE, Heitman SJ, Tsai WH, Wang J. James MJ. Effect of Treatment of Obstructive Sleep Apnea on Depressive Symptoms: Systematic Review and Meta-Analysis. PLOS Medicine. 2014; 11(11): e1001762. 


\section{INTERNATIONAL ARCHIVES OF MEDICINE

33. Edwards C, Mukherjee S, Simpson L, Palmer LJ, Almeida OP, Hillman DR. Depressive symptoms before and after treatment of obstructive sleep apnea in men and women. J Clin Sleep Med 2015; 11(9): 1029-38.

34. Lee SA, Han SH, Ryu HU. Anxiety and its relationship to quality of life independent of depression in patients with obstructive sleep apnea. J Psychosom Res. 2015; 79(1): 32-6.

35. Amdo T, Hasaneen N, Gold MS, Gold AR. Somatic syndromes, insomnia, anxiety, and stress among sleep disordered breathing patients. Sleep Breath. 2016; 20(2): 759-68.

36. Hayley AC, Williams LJ, Venugopal K, Kennedy GA, Berk M, Pasco JA. The relationships between insomnia, sleep apnoea and depression: findings from the American National Health and Nutrition Examination Survey, 2005-2008. Aust N Z J Psychiatry. 2015; 49(2): 156-70

37. Rezaeitalab F, MoharrariF, Saberi S, Asadpour H, and Rezaeetalab F. The correlation of anxiety and depression with obstructive sleep apnea syndrome. J Res Med Sci. 2014; 19(3): 205-210.

38. Lee W, Lee SA, Ryu HU, Chung YS, Kim WS. Quality of life in patients with obstructive sleep apnea: Relationship with daytime sleepiness, sleep quality,depression, and apnea severity. Chron Respir Dis. 2016; 13(1): 33-9.
39. Cai SJ, Chen R, Zhang YL, Xiong KP, Lian YX, Li J, Shen JC, Liu CF. Correlation of Epworth Sleepiness Scale with multiple sleep latency test and its diagnostic accuracy in assessing excessive daytime sleepiness in patients with obstructive sleep apnea hypopnea syndrome. Chin Med J (Engl). 2013; 126(17): 324550.

\section{Publish in International Archives of Medicine}

International Archives of Medicine is an open access journal publishing articles encompassing all aspects of medical science and clinical practice. IAM is considered a megajournal with independent sections on all areas of medicine. IAM is a really international journal with authors and board members from all around the world. The journal is widely indexed and classified Q1 in category Medicine. 